\title{
Transcriptomic Profiling of Fruit Development in Black Raspberry Rubus coreanus
}

\author{
Qing Chen $\mathbb{D}^{1},{ }^{1}$ Xunju Liu $\mathbb{D},{ }^{1}$ Yueyang Hu $\mathbb{D},{ }^{1}$ Bo Sun $\mathbb{D},{ }^{1}$ Yaodong Hu, ${ }^{2}$ Xiaorong Wang $\mathbb{D},{ }^{3}$ \\ Haoru Tang $\left(\mathbb{D},{ }^{1}\right.$ and Yan Wang $\mathbb{C}^{3}$ \\ ${ }^{1}$ College of Horticulture, Sichuan Agricultural University, Chengdu, Sichuan 611130, China \\ ${ }^{2}$ Science and Technology Management Division, Sichuan Agricultural University, Chengdu, Sichuan 611130, China \\ ${ }^{3}$ Institute of Pomology and Olericulture, Sichuan Agricultural University, Chengdu, Sichuan 611130, China \\ Correspondence should be addressed to Yan Wang; wangyanwxy@163.com
}

Received 6 September 2017; Revised 9 February 2018; Accepted 20 February 2018; Published 1 April 2018

Academic Editor: Marco Gerdol

Copyright (C) 2018 Qing Chen et al. This is an open access article distributed under the Creative Commons Attribution License, which permits unrestricted use, distribution, and reproduction in any medium, provided the original work is properly cited.

\begin{abstract}
The wild Rubus species R. coreanus, which is widely distributed in southwest China, shows great promise as a genetic resource for breeding. One of its outstanding properties is adaptation to high temperature and humidity. To facilitate its use in selection and breeding programs, we assembled de novo 179,738,287 $R$. coreanus reads ( $125 \mathrm{bp}$ in length) generated by RNA sequencing from fruits at three representative developmental stages. We also used the recently released draft genome of $R$. occidentalis to perform reference-guided assembly. We inferred a final 95,845-transcript reference for $R$. coreanus. Of these genetic resources, 66,597 $(69.5 \%)$ were annotated. Based on these results, we carried out a comprehensive analysis of differentially expressed genes. Flavonoid biosynthesis, phenylpropanoid biosynthesis, plant hormone signal transduction, and cutin, suberin, and wax biosynthesis pathways were significantly enriched throughout the ripening process. We identified 23 transcripts involved in the flavonoid biosynthesis pathway whose expression perfectly paralleled changes in the metabolites. Additionally, we identified 119 nucleotide-binding site leucine-rich repeat (NBS-LRR) protein-coding genes, involved in pathogen resistance, of which 74 were in the completely conserved domain. These results provide, for the first time, genome-wide genetic information for understanding developmental regulation of $R$. coreanus fruits. They have the potential for use in breeding through functional genetic approaches in the near future.
\end{abstract}

\section{Introduction}

The genus Rubus L. comprises $900-1000$ species and has a worldwide distribution (excluding Antarctica) with various climatic adaptations [1]. Plants used in fruit production are mainly from two subgenera, Rubus and Idaeobatus. Blackberries and raspberries are the most commonly cultivated fruits in these two subgenera. They are deemed functional fruits, mainly due to being rich sources of healthpromoting antioxidant or "nutraceutical" compounds (i.e., anthocyanins, phenolics, and ellagic acid) in fresh fruits [2] and anticancer properties in fruit extracts [3]. Historically, they have been used in traditional Chinese medicine and are mentioned in the Compendium of Materia Medica (Bencao Gangmu) compiled by Li Shizhen (1518-1593) during the Ming Dynasty. Chinese or Korean black raspberry $R$. coreanus Miq., in the subgenus Idaeobatus, is named for the dark red (or black) color of its fruits when mature. Earlier investigations found that black raspberry fruits contained higher concentrations of the nutritional ingredients mentioned above than either red raspberry or blackberry [4]. However, Chinese black raspberry is not as popular as the other two species as much less effort is given to its cultivation and there is only a limited choice of cultivars available. After a thorough investigation of the biochemical components in fruit [5], researchers from South Korea provided the first transcriptome analysis of what they believed to be $R$. coreanus [6]. Unfortunately, contrary to what is reported in their paper [7], the species they studied was the commercially grown North American black raspberry 
$R$. occidentalis, often confused with $R$. coreanus. In 2016, the first draft genome for $R$. occidentalis, $243 \mathrm{Mb}$ in size, became publicly available [8]. It is the most useful Rubus genetic resource to date.

$R$. coreanus has been used as a valuable genetic donor in several Rubus-breeding programs $[9,10]$ because of its outstanding disease resistance and high productivity. $R$. coreanus cultivars are also commercialized in South Korea [11]. The lack of a genetic reference for $R$. coreanus has become a barrier to application of modern breeding techniques, such as marker-assisted selection and transgenic strategies. Within the past ten years, we have made a comprehensive study of Rubus species in China, mostly those endemic to the country, focusing especially on wild species distributed in the south [12]. The relatively high temperature and humidity of the area may have led to the development of unique disease resistance characteristics in these Rubus species. Given that viral and fungal diseases have become one of the main threats hindering the development of commercial cultivation of Rubus [13], exploring underlying mechanisms for disease resistance and finding new candidate genetic resources could facilitate selection and breeding.

Fruit maturation is a complex process involving genetic regulation closely linked with environmental signaling of palatability. Pigment deposition and the resulting color change, and sugar accumulation, usually coupled with depletion of titratable acid leading to formation of specific sensory traits, are common signs of fruit ripeness [14]. Dissection of the intrinsic genetic changes of fruit maturation could bring new insights into understanding the consequences of application of a particular agronomic practice at a particular time. For example, Fragaria $\times$ ananassa has traditionally been classified as nonclimacteric because its ripening process is not governed by ethylene. However, global analysis of transcriptome data and the ethylene response factor (ERF) gene family has identified involvement of ethylene in ripening of the receptacle at specific tissue/developmental stages [15]. Moreover, unveiling the gene expression atlas of fruit maturation could enable greater understanding of biosynthesis of bioactive compounds, a necessary step toward breeding new varieties for health benefits. Considerable effort has been made in this regard for fruits such as grapes [16], blueberries [17], and tomatoes [18]; in comparison, very little effort has been made for Rubus species.

Therefore, as a first step towards understanding gene expression during fruit ripening in $R$. coreanus, this study presents results of a comprehensive analysis of transcriptome data from fruits at three representative developmental stages. Both de novo and reference-guided assembly were carried out to maximize the possibility of finding potential transcripts. We also investigated the genes for long noncoding RNAs. Differentially expressed genes, specifically, (1) genes leading to flavonoid biosynthesis and (2) plant nucleotidebinding site leucine-rich repeat (NBS-LRR) genes, which contribute to biotic and abiotic stress responses, were analyzed. It is hoped that exploration of the genetics of $R$. coreanus may prove to be a profitable endeavor by providing valuable information for Rubus breeding.

\section{Results and Discussion}

2.1. Transcriptome Sequencing and Sequence Assembly. Although Rubus species are some of the most popular functional fruits in the world, it is only recently that genomic resources have become available for the genus [8]. In Hyun et al.'s study of R. occidentalis (which they mistook for R. coreanus [6]) from the perspective of fruit morphology and phenological traits [7], transcriptome analysis involved mRNA isolated only from fruits sampled 20 days after anthesis at an intermediate stage of ripening [6]. This may have underestimated the genetic information for the species. In the present study, 179.74 million $125 \mathrm{bp}$ paired-end raw reads were generated from fruit libraries of three developmental stages. After trimming adapter-related reads and filtering low-quality reads, 65.27 million bases were subjected to error correction. Finally, 174.79 million reads comprising 43.7 gigabases were used to assemble the reference. In total, 78.80 million bases were assembled into 95,845 transcripts, with an N50 length of $1242 \mathrm{bp}$ (Table 1). The generated 125 bp paired-end reads are available at NCBI Sequence Read Archive SRR6001072 to SRR6001077 associated with BioProject PRJNA401210.

To assess the quality of the assembly further, Bowtie (v2.2.9) [19] was employed to align all reads back to the reference. Of the reads, $83.66 \%$ could be aligned, with $64.17 \%$ aligning concordantly and uniquely to the final version of the reference. In contrast, only $52.97 \%$ of the total reads could be aligned to the genome-guided assembly, indicating high divergence between $R$. coreanus and R. occidentalis. This result may partly explain the previously observed phenomenon that although these two species are easy to cross, the F1 progenies are completely sterile [20]. Therefore, it is reasonable that a proportion of our transcripts could not be mapped to the reference. In addition, when evaluated against the complete 1440 plant-specific orthologs in the Benchmarking Universal Single-Copy Orthologs (BUSCO) database [21], the largest proportion of the assembly (95.3\%) was complete, with only $27(1.9 \%)$ fragmented and 41 (2.8\%) missing orthologs. These results indicate high completeness of our assembly.

Taking expression values into consideration, we plotted the transcripts per million (TPM) distribution patterns of all transcripts (Figure 1(a)). Predominant portions of the transcripts were at low abundance. If using three TPM as a threshold, each fraction of 39,039 transcripts could be viewed as from one genuine gene. This number is within the range of gene numbers from the $R$. occidentalis genome project [8]. Taking this read coverage information before abundance filtering, the N50 value for the top Ex\% transcripts was calculated (Figure 1(b)). The maximum N50 value ( $2142 \mathrm{bp}$ ) was reached when taking $96 \%$ of the upper expressed gene products.

2.2. Functional Annotations for the R. coreanus Transcriptome. Annocript pipeline [22] was employed to annotate transcripts and coding peptides. Searches for homologous counterparts in the manually annotated, nonredundant protein sequence database Swiss-Prot (SP) and the subset UniProt 
TABLe 1: Overview of the assembly.

\begin{tabular}{lccc}
\hline & Rubus occidentalis genome guided & De novo & Final reference \\
\hline Total number of transcripts & 47,239 & 296,591 & 95,845 \\
Total nucleotides & $80,446,066$ & $214,031,901$ & $78,800,996$ \\
Average length (bp) & 1703 & 813 & 822 \\
Minimum length (bp) & 102 & 201 & 102 \\
Maximum length (bp) & 21,369 & 14,054 & 17,356 \\
N50 (bp) & 2496 & 1603 & 1242 \\
\hline
\end{tabular}

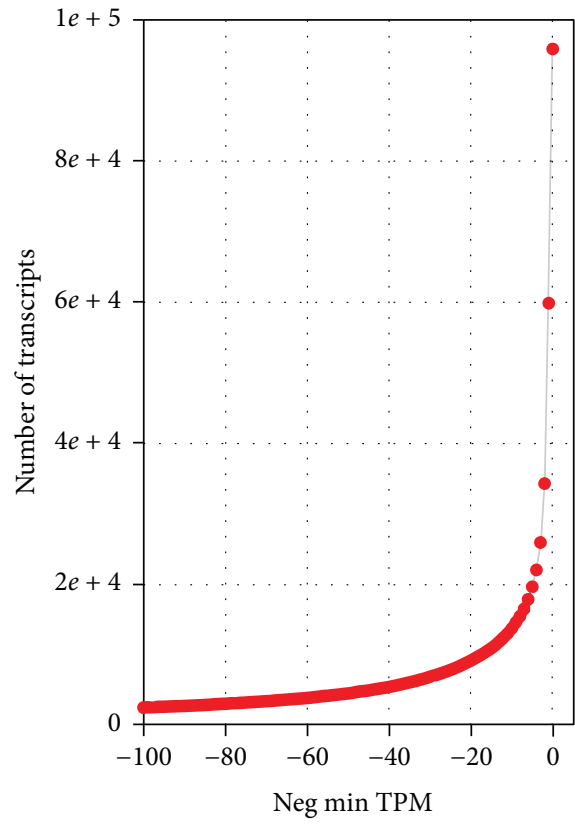

(a)

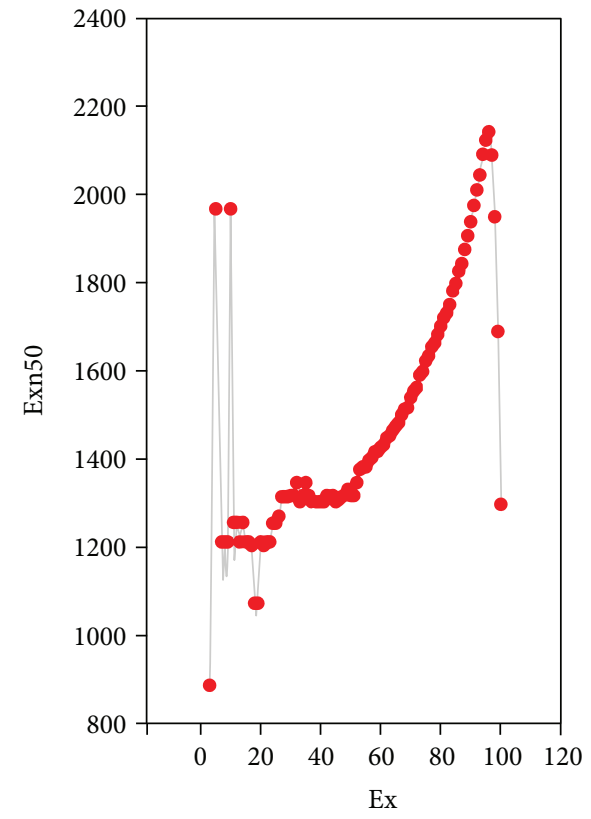

(b)

Figure 1: Expression statistics for all transcripts (a) and ExN50 distribution of the assembly (b). Neg min TPM in (a) indicates the negative value of a given minimum expression level as transcripts per million (TPM) reads. Ex indicates that $x \%$ of the assembled transcript nucleotides can be found in contigs that are at least of ExN50 length.

Reference Clusters Uniref90 (UF90) database by the blastx algorithm resulted in 47,090 (49.99\%) of the raw transcripts generating hits in SP and 66,520 (69.40\%) of the transcripts with homologs in UF90. More specifically, $15.60 \%$ of the SP hits and $25.43 \%$ of the UF90 hits were covered over $80 \%$ by the enquiry sequences. In the case of gene ontology (GO) assignment, 51,520 (53.758\%) transcripts could be classified into Biological Process $(28,547)$, Cellular Component $(31,728)$, or Molecular Function $(41,971)$ categories. Searches in the Kyoto Encyclopedia of Genes and Genomes (KEGG) Orthology (KO) database against related plants resulted in $\mathrm{KO}$ identifiers of 42,769 of the transcripts being assigned to the corresponding pathways. Through PORTRAIT noncoding potential evaluation using a new support vector machine-based algorithm [23], 2178 long noncoding RNA- (lncRNA-) coding genes were also discovered. Taken together, these results suggest that the de novo assembled reference covered a wide range of Rubus genetic information, which provides a valuable resource for facilitating the discovery of novel genes involved in specific physiological and developmental processes.

2.3. Analysis of Differential Gene Expression across the Three Developmental Stages of $R$. coreanus Fruit. We mapped all reads from each fruit developmental stage and estimated transcript counts against the reference using the RSEM method [24]. Transcripts with less than three TPM across the three stages were filtered in the subsequent differentially expressed gene (DEG) assay based on the above analysis. Three different expression analysis packages were used for DEG detection: (1) DESeq2 [25], which uses a Wald test; (2) edgeR [26], which uses a likelihood ratio test; and (3) limma-voom [27], which uses a moderated $t$-test, to compare expression differences between fruit stages. In the consensus results, 211 transcripts were expressed differentially in red fruits compared to green fruits. Among these genes, 49 were downregulated and 162 were upregulated. Between black (mature) and red fruit stages, 1141 genes were upregulated 
and 1423 downregulated. Variation in expression was observed in 2363 genes between black and green fruits. Although the strict criteria we used in this analysis may overlook other gene products, they can be viewed as generating the most reliable DEGs.

$\mathrm{GO}$ and $\mathrm{KO}$ enrichment analyses were carried out to consider more closely these differentially expressing genes. When testing for GO terms detected from differentially expressed genes in green versus red, red versus black, and green versus black fruits, no significantly enriched genes were found by GOEAST (http://omicslab.genetics.ac.cn/GOEAS T/tools.php). However, several biological pathways were found to be significantly perturbed. Sixteen pathways were enriched across the whole fruit developmental process, including those of genes involved in flavonoid biosynthesis, phenylpropanoid biosynthesis, plant hormone signal transduction, and cutin, suberin, and wax biosynthesis, among others (Figures 2-4). In addition to these commonly impacted pathways, alterations in "degradation of aromatic compounds" and "MAPK signaling pathway - plant" were detected specifically in the early stages (change from small green to red fruit). In contrast, "bisphenol degradation" and "polycyclic aromatic hydrocarbon degradation" pathways were affected in the later stages (change from red to fully ripe black fruit). Fruit ripening is a process of highly coordinated and genetically programed physiological, biochemical, and organoleptic changes in the reproductive organs. In Rubus fruits, predominant changes in ripening include (1) depolymerization of carbohydrates, specifically, degradation of starches into sucrose and then into glucose and fructose; (2) decrease in organic acids, including amino acids; (3) production of volatile compounds, such as alcohols and aldehydes; and (4) accumulation of anthocyanins but depletion of cinnamic, ferulic, protocatechuic, and vanillic acids and epicatechin [5]. These changes may be evident in the metabolic pathway profiling in our study. The starch and sucrose metabolism pathway, significantly enriched in the two early stages of fruit ripening, adding to the degradation of aromatic compounds, can lead to the formation of special flavor and aroma of ripening Rubus fruits. A mixture of compounds, including ketones, alcohols, esters, and mainly terpenoids, constitutes the volatile flavor of most, if not all, fruits [28]. Some Rubus species have a special aroma to their fruit, but some do not [29]. Degradation of aromatic compounds could have a partial impact on these aroma volatiles. Another obvious sign of maturation of soft fruits is the decrease in firmness, which is the result of degradation of cell wall components and/or loss of integrity of the cell cuticular/wax layer $[30,31]$. In strawberries, cell wall disassembly is characterized by solubilization of pectins, slight depolymerization of covalently bound pectins, and loss of galactose and arabinose, as well as a reduction in hemicellulose content [32]. Pectin content of mature fruit reduced dramatically in two raspberry cultivars, "Glen Clova" and "Glen Prosen" [33]. Further examination of $R$. idaeus cell wall fraction indicated that fruit ripening was associated with increased solubilization of pectin first and then depolymerization at the last stage [34]. In support of this, DEGs for cutin, suberin, and wax biosynthesis were found to be significantly enriched across the three fruit-ripening stages in our study. Only two DEGs (omegahydroxypalmitate O-feruloyl transferase and peroxygenase) were common to all three stages. Progressive modulation of these particular genes may be the molecular basis of programing of the fruit-softening process.

\subsection{Flavonoid Biosynthesis Genes and Their Expression.} Anthocyanin, the most important metabolite in flavonoid production, is an essential nutritional component in raspberry fruits and their products $[35,36]$. In $R$. coreanus, cyanidin-3-glucoside, cyanidin-3-rutinoside, and pelargonidin-3glucoside have been recognized as the major anthocyanins [35]. Anthocyanins are first detected in green-red fruit but increase at the greatest rate to the highest amount in the last developmental stage [5]. The same trend has been observed for flavonols such as quercetin-glucuronide and quercetin3-O-rutinoside. In contrast, flavanols and proanthocyanidins are accumulated at the very beginning of fruit set [5]. All these compounds are final products typical of the flavonoid biosynthesis, anthocyanin, and flavonol synthesis pathways. In our study, both flavonoid and anthocyanin pathways were significantly enriched during fruit development. This is in accordance with findings for other fruits such as grapes [16] and bayberries [37]. Confirming our prediction from the KEGG pathway enrichment above, we were able to manually identify 23 transcripts involved in the flavonoid biosynthesis pathway leading to flavonols, anthocyanins, or proanthocyanidins. The expression of most of these transcripts perfectly parallels the changes in the metabolites (Figure 5). Among these genes, five have alternative transcripts/multigene members including two phenylalanine ammonia-lyases (PAL), six 4-coumarate:CoA ligases (4CL), three chalcone synthases (CHS), two flavonol synthases (FLS), and two dihydroflavonol 4-reductases (DFR). Major players among the transcripts from the same gene/multigene could be identified from expression patterns. For example, among the six 4CL transcripts, transcript_52752 may be the key actor, whose abundance increased highly in parallel with fruit maturation. In comparison, although their roles could not be ruled out, most other transcripts of $4 C L$ exhibited very low levels of expression throughout the three fruit developmental stages. Functional diversification could be deduced from the results if multigene copies existed. Examples include PAL (transcript_21400 and transcript_22918) and DFR (transcript_1703 and transcript_61515). One member of $P A L$ or DFR had a completely opposite expression mode compared to the other (Figure 5). In strawberries, the two copies of DFR have different substrate affinities, exerted at different stages for producing different types of anthocyanin [38]. Therefore, the function of the DFRs isolated in R. coreanus needs further investigation. Also noteworthy is the absence of the $F 3^{\prime} 5^{\prime} \mathrm{H}$ gene in the transcriptome, which implies that the synthesis of delphinidin-derived anthocyanins is blocked in $R$. coreanus fruits.

2.5. Identification and Abundance Estimation of NBS-LRREncoding Genes. Fungal and viral diseases are two worldwide threats to commercial cultivation of Rubus. Given the requirement for reducing pesticide use, cultivars with robust 


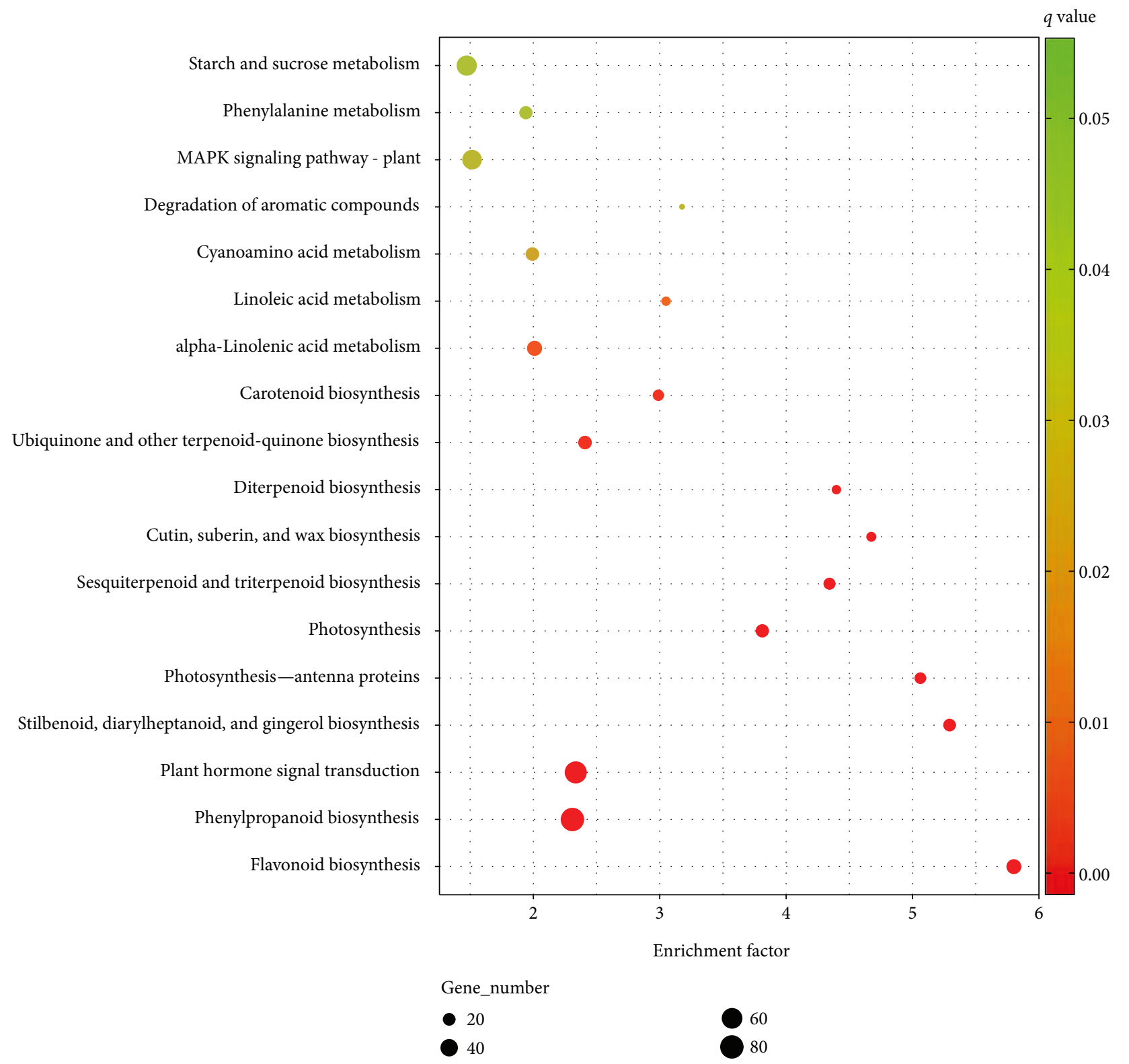

FIgURe 2: Pathway enrichment of differentially expressed genes between green and red Rubus coreanus fruits.

disease resistance become increasingly important. Fungal pathogens attack every part of Rubus, including the roots, canes, leaves, and fruits. Several fungal diseases can cause pre- or postharvest fruit rot in raspberries, leading to a short shelf life and limited sales of fresh fruit to distant markets. Gray mold (Botrytis cinerea Pers.:Fr.) is the most serious pathogen of fruit. Variation in susceptibility to it has been observed in fruits from different raspberry cultivars [39]. It is well documented that $R$. coreanus derivatives have strong resistance to cane diseases caused by Elsinoë veneta, Didymella applanata, and B. cinerea [40]. $R$. coreanus has also been recommended as a resource for promoting fruit rot resistance $[10,41,42]$. Proteins that contain a nucleotidebinding site and leucine-rich repeat (NBS-LRR) domains consist of the largest class of known plant resistance (R) gene products, conferring resistance to a diverse spectrum of pathogens $[43,44]$. Recent advances have revealed that NBS-LRR R proteins are able to inhibit B. cinerea development [45]. In the family Rosaceae, NBS-LRR-coding genes form a large proportion of the genome, from $1.05 \%$ in strawberries to $1.52 \%$ in peaches [46]. However, except of a few studies, the availability of $\mathrm{R}$ gene resources in Rubus species is limited. Samuelian et al. [47] characterized 75 LRR genes from $R$. idaeus using degenerate primers designed from other Rosaceae species. Afanador-Kafuri and colleagues [48] obtained 47 LRR proteins using a similar strategy from Colombian Rubus genotypes. In a further exploration of our transcriptome data, initial screening via hmmsearch of the new reference uncovered 411 candidate NBS-encoding transcripts. Thereafter, through domain hunting, 119 NBSLRR-domain-coding transcripts were identified, among which 74 had hits in the completely conserved NBS domain 


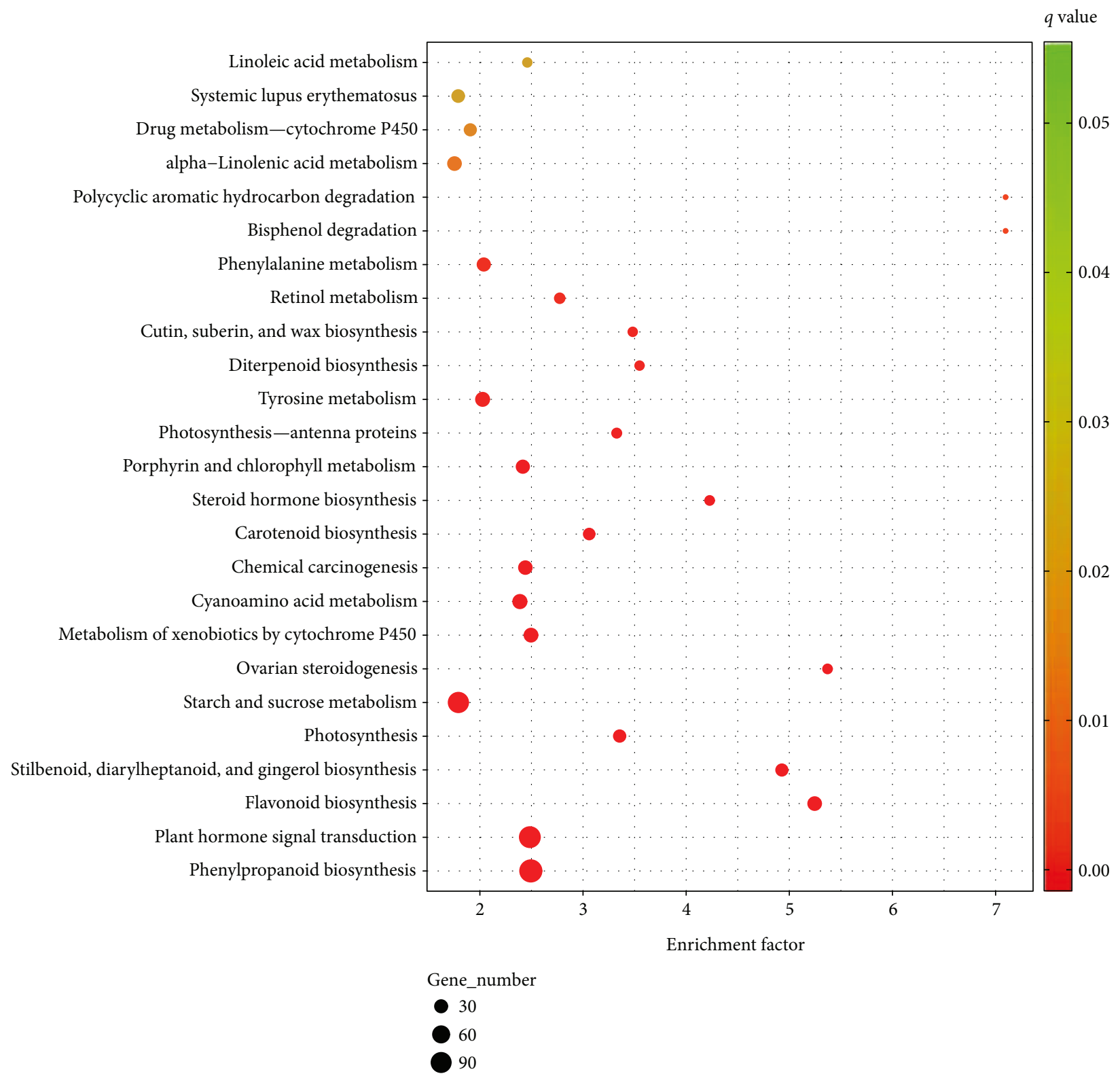

FIGURE 3: Pathway enrichment of differentially expressed genes between red and black Rubus coreanus fruits.

(Supplementary Table 1). We believe our resources greatly enrich the genetic information for Rubus breeding. Most of these plant resistance protein-coding genes have low abundance (less than five) estimated as trimmed mean of $M$ values (TMM). This appears reasonable because a very high expression of $\mathrm{R}$ proteins could bring lethal effects to plant cells [49]. Twenty-four NBS transcripts are presented in Figure 6. Two of the transcripts show relatively high expression values (transcript_24284 and transcript_72010) in fruits at almost all three stages. Transcript_47133, with the highest abundance, functions mainly in the last stage, when fruits are fully ripe and are more vulnerable to pathogen attack. Closer examination of this resistance gene found that it is in the class of NBS-LRR (NL) proteins lacking additional N-terminal domains. Its closest ortholog in $R$. occidentalis is the gene Bras_G19818, which shares
$62.58 \%$ sequence identity. Some of the NBS-LRR genes have tissue-specific expression properties [50] and can even confer different resistance reactions with different alleles from the same gene [51]. These three highly expressed or stage-specific factors could be interesting candidates for more detailed investigation in the future.

2.6. Cloning and Real-Time Quantitative PCR (RT-qPCR) Validation of Representative Genetic Information. Seven representative genes (ANR, CER, CHI, CYP86B1, DFR, GPAT, and MYB44), which encode key enzymes or regulators involved in anthocyanin/proanthocyanidin biosynthesis, amino acid metabolism, or plant cell wall wax formation, were successfully cloned and validated by sequencing. All these gene sequences corresponding to the full length of coding sequence with various lengths of $5^{\prime}$ or $3^{\prime}$ untranslated 


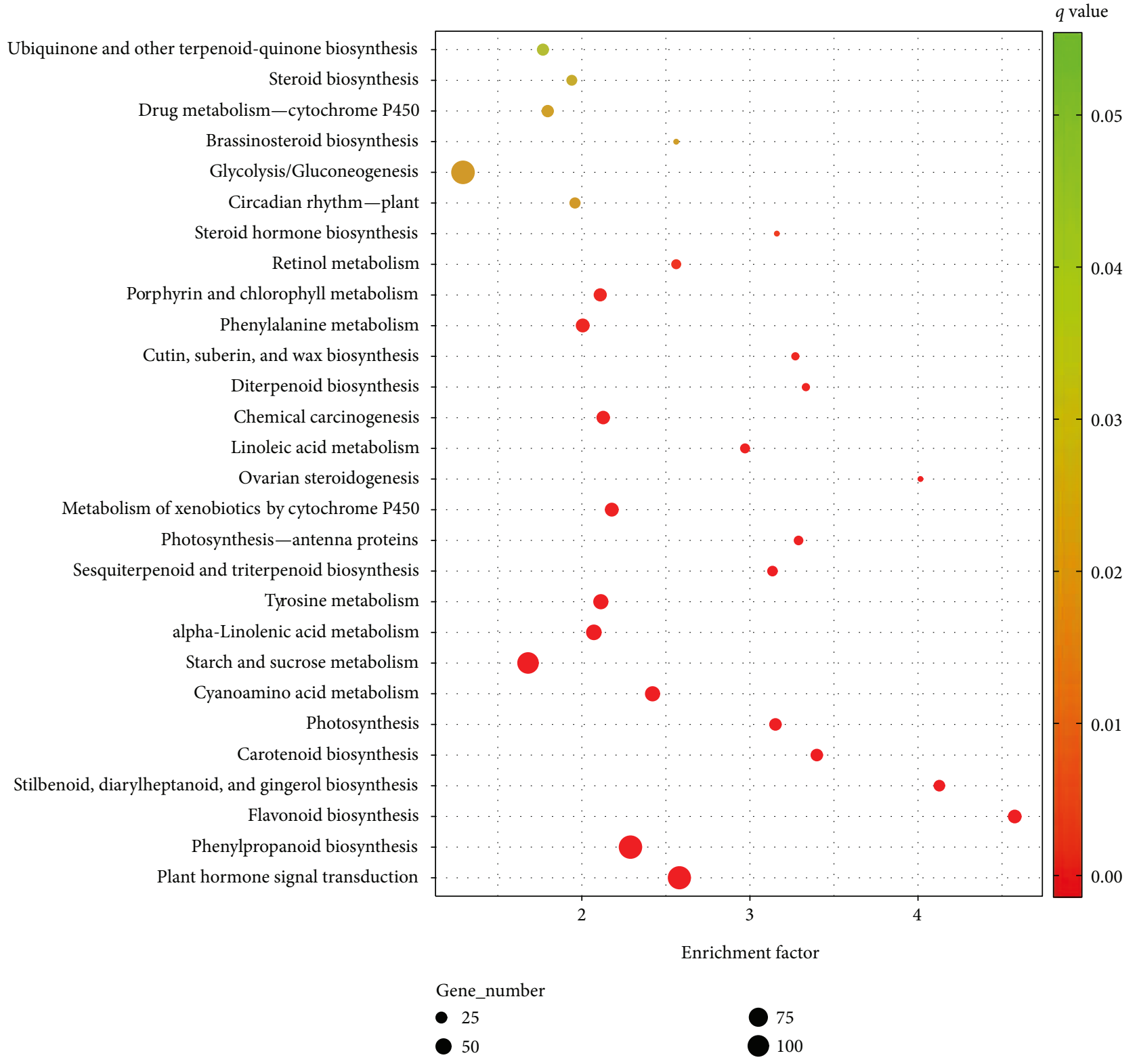

Figure 4: Pathway enrichment of differentially expressed genes between green and black Rubus coreanus fruits.

region (UTR) were identical to those deduced from the RNAseq results. Similar expression patterns between RNA-seq and RT-qPCR were also observed (Figure 7), thus further validating the RNA-seq expression data.

\section{Conclusions}

This is the first transcriptomic profile, through RNA-seq investigation of sequence and transcript abundance, for $R$. coreanus. The transcriptomic analysis provides, for the first time, a 95,845-transcript reference for the species. Of these genetic resources, $69.5 \%$ were annotated. Differentially expressed genes in fruit developmental stages were mainly involved in flavonoid biosynthesis, plant cell wall formation, and aroma compound degradation. We identified 23 transcripts involved in the flavonoid biosynthesis pathway whose expression perfectly paralleled changes in the metabolites. Additionally, we identified 119 nucleotidebinding site leucine-rich repeat (NBS-LRR) protein-coding genes, involved in pathogen resistance, of which 74 were in the completely conserved domain. We believe that our study provides useful genetic information for Rubus breeding.

\section{Materials and Methods}

4.1. Sample Collection and RNA Preparation. Fruits of R. coreanus $(2 n=2 x=14)$ [12] were collected from the wild at Ya'an city, Sichuan province $\left(29^{\circ} 58^{\prime} 24.5^{\prime \prime} \mathrm{N}, 103^{\circ} 00^{\prime} 18.7^{\prime \prime} \mathrm{E}\right)$. Fruit set occurs in April and fruits mature in mid-June in this area. Fruits of three representative stages of ripening (green, red, and mature black) were harvested in 2015. For each stage, a total of about 30 fruits from no more than three canes 

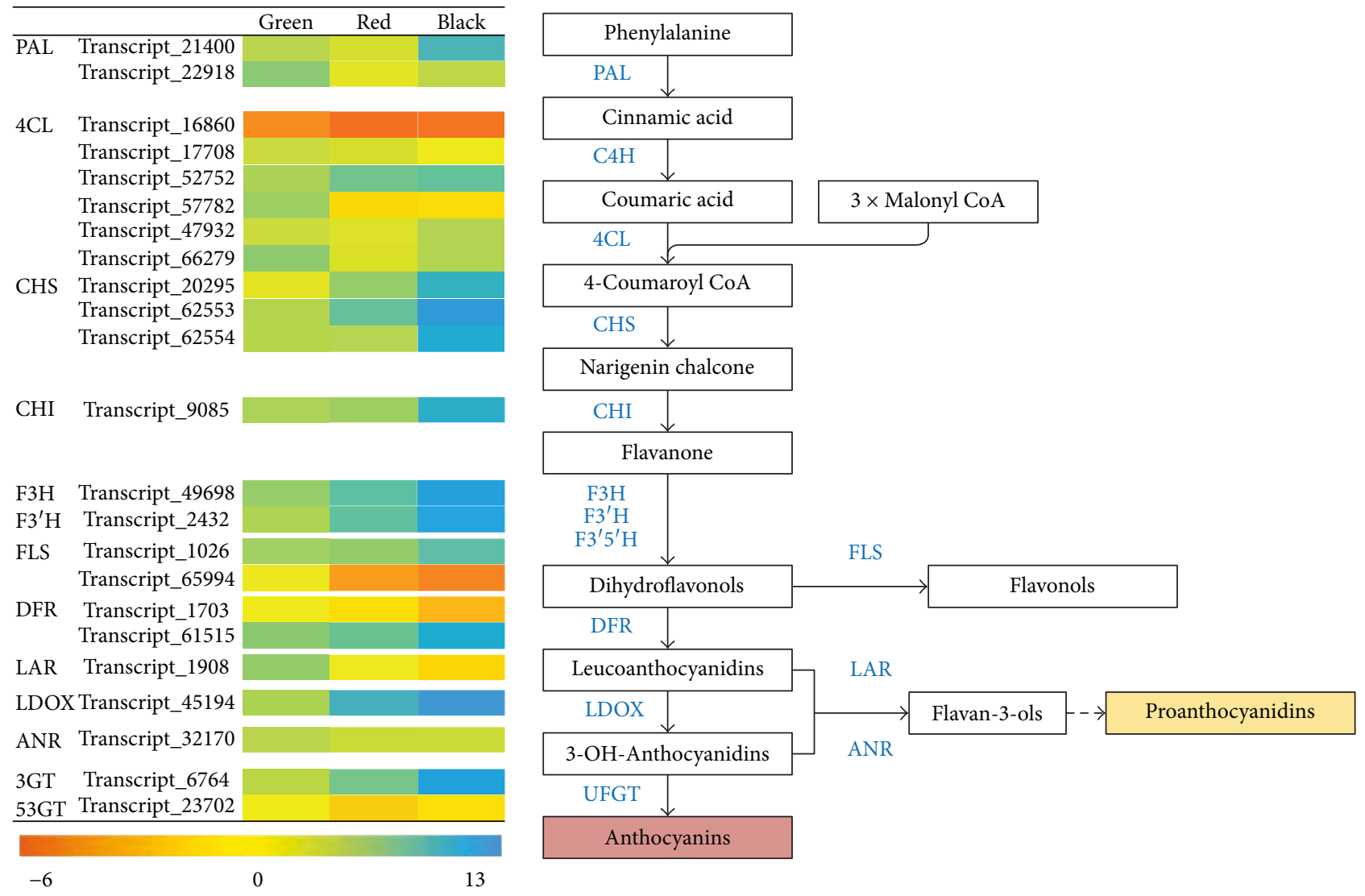

FIGURE 5: Flavonoid-synthesis-associated transcripts and their expression patterns during fruit ripening. Expression values are presented as $\log$ 2-transformed trimmed mean of $M$ value (TMM) derived from edgeR analysis. PAL: phenylalanine ammonia-lyase; 4CL: 4-coumarate:CoA ligase; CHS: chalcone synthase; CHI: chalcone flavanone isomerase; F3H: flavanone 3-hydroxylase; F3' H: flavonoid 3'-hydroxylase; F3'5'H: flavonoid 3',5'-hydroxylase; FLS: flavonol synthase; DFR: dihydroflavonol 4-reductase; LDOX: leucoanthocyanidin dioxygenase; LAR: leucoanthocyanidin reductase; ANR: anthocyanidin reductase; 3GT: anthocyanin 3-O-glucosyltransferase; 53GT: anthocyanin 3,5-O-glucosyltransferase.

were collected in order to decrease background variation. They were frozen immediately in liquid nitrogen on collection and stored at $-80^{\circ} \mathrm{C}$ until use. Two biological replicated samples were collected for each stage due to the limited yield of fruits.

Total RNA was isolated by using a modified cetyltrimethylammonium bromide method [52]. Genomic DNA was eliminated by using RNase-free DNase I (TaKaRa, Dalian, China). After monitoring RNA integrity and purity on 1\% agarose gels and NanoPhotometer spectrophotometer (Implen, CA, USA), the Agilent 2100 Bioanalyzer system (Agilent Technologies, CA, USA), supplemented with RNA 6000 Nano Kit, was used to confirm the results. RNA concentration was measured using Qubit RNA Assay Kit in Qubit 2.0 Fluorometer (Life Technologies, CA, USA).

4.2. cDNA Synthesis, Library Construction, and Sequencing. As input material, $3 \mu \mathrm{g}$ of RNA per sample was used. Sequencing libraries were generated using NEBNext Ultra Directional RNA Library Prep Kit for Illumina (NEB, USA) according to the manufacturer's instructions. Briefly, mRNA was purified from total RNA using poly(T) oligo-attached magnetic beads. Fragmentation was carried out using divalent cations under elevated temperature in NEBNext First Strand Synthesis reaction buffer (5x). First-strand cDNA was synthesized with random hexamer primer and M-MuLV Reverse Transcriptase (RNase H). Second-strand cDNA was synthesized by DNA polymerase I and RNase H. Remaining overhangs were blunted via exonuclease/polymerase activities. After adenylation of $3^{\prime}$ ends of DNA fragments, NEBNext Adaptors with a hairpin loop structure were ligated. AMPure XP system (Beckman Coulter, Beverly, USA) was used to purify cDNA fragments selectively at the correct size. Then, $3 \mu \mathrm{l}$ of USER Enzyme (NEB, USA) was used with size-selected, adaptor-ligated $\mathrm{cDNA}$ at $37^{\circ} \mathrm{C}$ for $15 \mathrm{~min}$ followed by $5 \mathrm{~min}$ at $95^{\circ} \mathrm{C}$. PCR was then performed with Phusion High-Fidelity DNA Polymerase, universal PCR primers, and index primers. Finally, the products were purified (AMPure XP system) and library quality was assessed on the Agilent 2100 Bioanalyzer system. Clustering and sequencing were carried out with the prepared libraries by Novogene (Beijing, China) using the Illumina HiSeq 2500 platform.

4.3. Transcriptome Assembly, Annotation, and Differential Expression and Enrichment Analysis. The raw reads were 


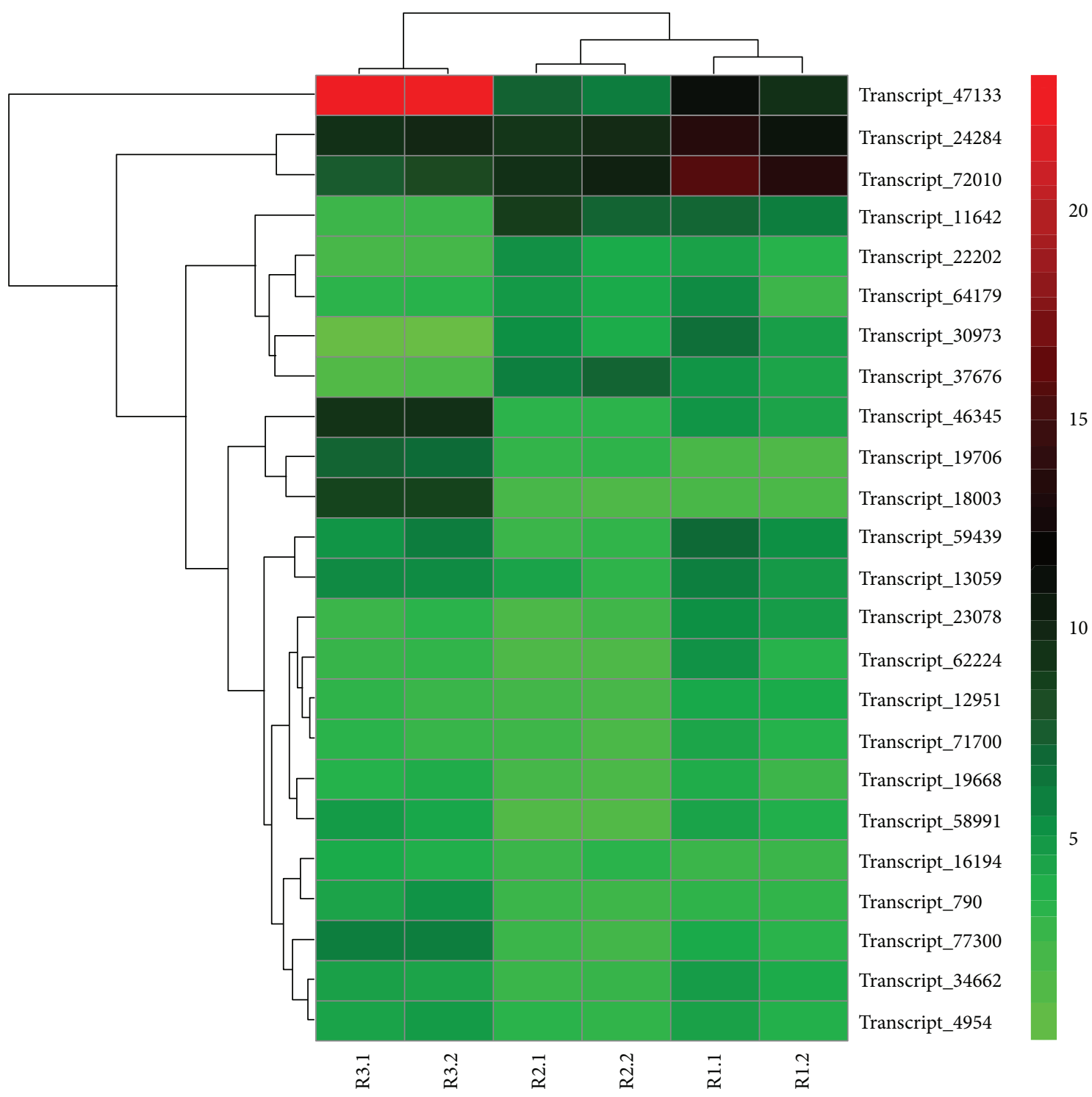

FIgURE 6: Heat map of the top 24 NBS-LRR genes expressed in Rubus coreanus. Normalized expression values are presented as trimmed mean of $M$ value (TMM) derived from the edgeR package.

cleaned by removing adapter sequences and ambiguous reads (with " $N$ " $>10 \%$ ). Low-quality bases were trimmed, and reads that were too short were filtered through Trimmomatic (LEADING:3 TRAILING:3 SLIDINGWINDOW:4:15 MINLEN:50) [53], as were the corresponding read pairs. After trimming/filtering low-quality reads, SEECER (v0.1.3) [54] was used for error correction. All downstream analyses were based on high-quality clean data.

To facilitate the use of the recently published North American black raspberry genome information, we adopted the strategies of genome-guided transcript expression analysis by using the protocol of Trapnell et al. [55]. All reads were first mapped to the $R$. occidentalis genome (v1.0) with TopHat2 (allowing two bases of mispairing and multiple hits $\leq 20$ ) and then assembled by using the Cufflinks suite with default parameters.

To evaluate divergence between $R$. coreanus and $R$. occidentalis, we also carried out de novo assembly of transcripts.
Trinity (v2.2.0) [56] was used with default parameters except that the minimum contig length was set to $200 \mathrm{bp}$, reads were first normalized with maximum coverage 50 before putting in the assembly pipeline, and k-mer coverage was set to a minimum level of two. Redundancy in the de novo transcriptome was minimized with CD-HIT-EST (v4.6.4) [57] using an identity cutoff at 0.99. EvidentialGene tr2aacds pipeline [58] was used to combine both genome-guided and de novo assemblies. Nonredundant transcripts were also obtained. To evaluate the quality of the reference, all assemblies were searched against BUSCO for plants [21].

All reads in each sample were mapped back to the transcriptome using Bowtie 2 [19] (default parameters used, but end to end, allowing two bases of mispairing and multiple hits $\leq 20)$ and then used to estimate expression values for each transcript by RSEM [24]. Given that many of the very lowly expressed transcripts could be questionable due to 


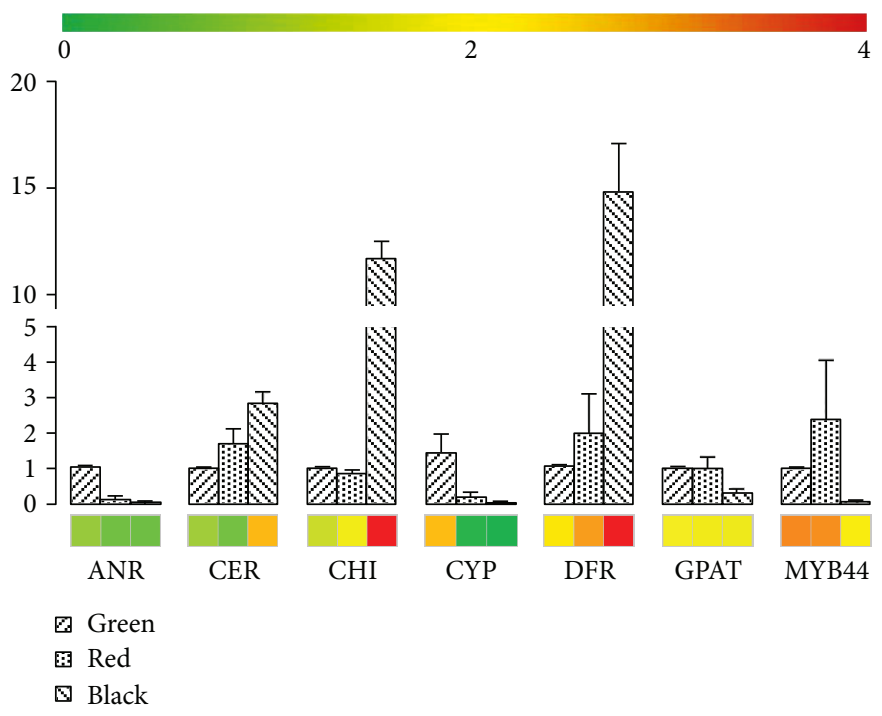

FIGURE 7: Similarities in expression patterns of seven genes between RNA-seq (heat map) and RT-qPCR (bar plot). ANR: anthocyanidin reductase; CER: ECERIFERUM; CHI: chalcone flavanone isomerase; CYP: cytochrome P450; DFR: dihydroflavonol 4-reductase, (transcript_61515 was chosen for DFR); GPAT: glycerol-3-phosphate acyltransferases. MYB44 is the transcript most resembling AtMYB44 in Arabidopsis thaliana.

our very high deep-sequencing coverage (exceeding 100x), we filtered the transcripts by setting transcripts per million (TPM) lower than three before conducting differential gene expression analysis.

Gene annotations were carried out according to the Annocript (v1.1.3) pipeline [21] using all assembled transcripts. We performed similarity searches through blastx against UniRef90 and Swiss-Prot (v201706, word_size $=4$; $\mathrm{e}$-value $=0.00001)$, rpsblast against the Conserved Domain Database (CDD) profiles (ftp://ftp.ncbi.nih.gov/pub/mmdb/ cdd/little_endian/Cdd_LE.tar.gz, e-value $=0.00001$, num_descriptions $=20$, and num_alignments $=20$ ), and blastn against Rfam and rRNAs (e-value $=0.00001$, num_descriptions $=1$, num_alignments $=1$, and num_threads $=4$ ). For each sequence, the best hit, if any, was chosen. For gene ontology (GO) functional classification, Enzyme Commission IDs were associated to the corresponding matches. KEGG Orthology (KO) assistant pathway assignment was implemented via KOBAS 3.0 [59] using the default parameters. The dna2pep tool implemented in the Annocript package [21] was used to identify the longest open reading frame (ORF) of each transcript. PORTRAIT software [23], which was developed for detecting noncoding RNA from poorly characterized species, was used to identify the noncoding potential of each query sequence by using a new support vector machine-based algorithm.

To investigate differential expression (DE) of transcripts, we used DESeq2 [25], which uses a Wald test; edgeR [26], which uses a likelihood ratio test; and limma-voom [27], which uses a moderated $t$-test to conduct pairwise comparison of the three fruit-ripening stages. Each of the comparisons was based on different statistical models. Differentially expressed genes were selected using $\log 2 \mathrm{FC} \geq 1$ or $\log \mathrm{FC} \leq-1$ and FDR (false discovery rate) $<0.01$ in the three methods. Consensus DE results were obtained by comparing the outcomes of the three methods, which were used to present the most reliable differentially expressed transcripts. These transcripts associated with their corresponding GO or $\mathrm{KO}$ annotations were tested against the whole transcriptome as background gene sets for enrichment analysis. GO categories were checked using GOEAST (http://omicslab.genetics.ac. cn/GOEAST/tools.php) with an FDR (Benjamini-Yekutieli method) value of $\leq 0.05$ as the cutoff to identify enriched terms by the hypergeometric test. Transcripts with a $\mathrm{KO}$ number were also tested by hypergeometric statistics to find significantly overperturbed pathways through a Perl inhouse script.

4.4. Expression Patterns of Genes Involved in Flavonoid Synthesis. From the gene differential expression analysis, flavonoid biosynthesis pathway genes appeared to be extremely perturbed in both green versus red fruits and red versus black fruits. We identified all genes involved in the pathway from the assembled reference and manually curated by blasting against the nonredundant protein database at the National Center for Biotechnology Information site, coupling the annotation from Annocript described above. Afterwards, expression patterns of these genes were presented as heat maps after $\log 2$ transformation of the among-sample normalized count values by using edgeR.

4.5. NBS-Encoding Gene Retrieval and Expression Analysis. Based on the Annocript-deduced peptide collection, we identified potential NBS-encoding genes using the procedures described by Arya et al. [60]. Specifically, the hidden Markov model (HMM) profile for the NBS domain (http://pfam.xfam. org/family/PF00931) was used to search against the complete set of the predicted $R$. coreanus proteins using hmmsearch in HMMER (v3.1b) [61] with e-value $<0.00001$. All the protein sequences identified were further subjected to CDD detection 
(https://www.ncbi.nlm.nih.gov/Structure/bwrpsb/bwrpsb.cgi) using 0.01 as a cutoff value to confirm the presence of NBS domains. Expression pattern analysis was carried out the same way as for flavonoid genes.

4.6. Cloning and RT-qPCR for Validation of Gene Expression Patterns. To verify the validity of the genetic information obtained, we selected seven representative genes (ANR, CER1, CHI, CYP, DFR, GPAT, and MYB44), which encode key enzymes involved in anthocyanin/proanthocyanidin biosynthesis, amino acid metabolism, plant cell wall wax formation, or stress response regulators. The deduced full coding sequences were cloned experimentally, and their expression values were determined using RT-qPCR. All the candidate sequences were amplified in a $20 \mu \mathrm{l}$ reaction mixture, containing $10 \mathrm{ng}$ first-strand cDNA, $10 \mathrm{pmol}$ each primer (Supplementary Table 2), and $10 \mu \mathrm{l} 2 \mathrm{x}$ PrimeSTAR HS premix (TaKaRa, Dalian, China). Following one cycle of 20 seconds at $98^{\circ} \mathrm{C}, 35 \mathrm{PCR}$ cycles of $10 \mathrm{~s}$ at $98^{\circ} \mathrm{C}, 10 \mathrm{~s}$ at $60^{\circ} \mathrm{C}$, and $90 \mathrm{~s}$ at $72^{\circ} \mathrm{C}$ were performed in the thermal cycler PTC200 (Bio-Rad, Hercules, CA). Amplified products were purified using E.Z.N.A. Gel Extraction Kit (Omega, GA, USA). The enriched PCR product was cloned into pEASYBlunt vectors (TransGen, Beijing, China) and transformed into JM109 competent Escherichia coli cells. Finally, positive clones were sequenced using the BigDye Terminator Cycle Sequencing Kit on an ABI PRISM 3730 automated DNA sequencer (Applied Biosystems, Foster City, CA, USA). For quantitative PCR, $10 \mu \mathrm{l}$ reaction mixture is composed of $5 \mu \mathrm{l}$ 2x SYBR Green mixture (TaKaRa, Dalian, China), $1 \mu \mathrm{l}$ diluted cDNA, and $1 \mu \mathrm{l}$ specific forward and reverse primer for each gene (Supplementary Table 1). The reaction was conducted on a CFX96 Real-Time PCR Detection System (Bio-Rad, US). Expression values were expressed as $2^{-\Delta \Delta C T}$ using beta-actin [52] as an internal control.

\section{Conflicts of Interest}

The authors declare that the research was conducted in the absence of any commercial or financial relationships that could be construed as a potential conflict of interest.

\section{Authors' Contributions}

Qing Chen and Yan Wang designed and supervised the experiment. Qing Chen and Xunju Liu conducted the experiment. Yueyang Hu assisted in RNA isolation. Xunju Liu and Yueyang $\mathrm{Hu}$ conducted the RT-qPCR analysis. Bo Sun and Xiaorong Wang helped in field plant identification and collection. Yaodong $\mathrm{Hu}$ and Haoru Tang provide guidance in bioinformatic analysis pipeline. Qing Chen and Yan Wang drafted and all authors revised the manuscript. Qing Chen and Xunju Liu contributed equally to this work.

\section{Acknowledgments}

The work was financially supported by the Basic Research Programs of Sichuan Province (2015JY0020) and the National Natural Science Foundation of China (31600232).
The authors also would like to thank the staff members at the computational division of the Institute of Pomology and Olericulture of Sichuan Agricultural University for computation assistance.

\section{Supplementary Materials}

Supplementary Table 1: domain identification and completeness of NBS-LRR proteins in Rubus coreanus fruits via CDD scan. Supplementary Table 2: primers used in the amplification and RT-qPCR analysis. (Supplementary Materials)

\section{References}

[1] M. M. Thompson, "Survey of chromosome numbers in Rubus (Rosaceae: Rosoideae)," Annals of the Missouri Botanical Garden, vol. 84, no. 1, pp. 128-164, 1997.

[2] R. Bobinaite, P. Viškelis, and P. R. Venskutonis, "Variation of total phenolics, anthocyanins, ellagic acid and radical scavenging capacity in various raspberry (Rubus spp.) cultivars," Food Chemistry, vol. 132, no. 3, pp. 1495-1501, 2012.

[3] N. P. Seeram, L. S. Adams, Y. Zhang et al., "Blackberry, black raspberry, blueberry, cranberry, red raspberry, and strawberry extracts inhibit growth and stimulate apoptosis of human cancer cells in vitro," Journal of Agricultural and Food Chemistry, vol. 54, no. 25, pp. 9329-9339, 2006.

[4] L. C. Torre and B. H. Barritt, "Quantitative evaluation of rubus fruit anthocyanin pigments," Journal of Food Science, vol. 42, no. 2, pp. 488-490, 1977.

[5] H.-S. Kim, S. J. Park, S.-H. Hyun et al., "Biochemical monitoring of black raspberry (Rubus coreanus Miquel) fruits according to maturation stage by ${ }^{1} \mathrm{H}$ NMR using multiple solvent systems," Food Research International, vol. 44, no. 7, pp. 1977-1987, 2011.

[6] T. K. Hyun, S. Lee, Y. Rim et al., "De-novo RNA sequencing and metabolite profiling to identify genes involved in anthocyanin biosynthesis in Korean black raspberry (Rubus coreanus Miquel)," PLoS One, vol. 9, no. 2, article e88292, 2014.

[7] J. Lee, M. Dossett, and C. E. Finn, "Mistaken identity: clarification of Rubus coreanus Miquel (Bokbunja)," Molecules, vol. 19, no. 7, pp. 10524-10533, 2014.

[8] R. VanBuren, D. Bryant, J. M. Bushakra et al., "The genome of black raspberry (Rubus occidentalis)," The Plant Journal, vol. 87, no. 6, pp. 535-547, 2016.

[9] V. H. Knight, D. L. Jennings, and R. J. McNicol, "Progress in the UK raspberry breeding programme," Acta Horticulturae, vol. 262, no. 262, pp. 93-104, 1989.

[10] C. Finn, J. R. Ballington, C. Kempler, H. Swartz, and P. P. Moore, "Use of 58 Rubus species in five north American breeding programmes-breeders notes," Acta Horticulturae, no. 585, pp. 113-119, 2002.

[11] S. H. Kim, H. G. Chung, and J. Han, "Breeding of Korean black raspberry (Rubus coreanus Miq.) for high productivity in Korea," Acta Horticulturae, no. 777, pp. 141-146, 2008.

[12] X. R. Wang, Y. Liu, B. F. Zhong et al., "Cytological and RAPD data revealed genetic relationships among nine selected populations of the wild bramble species, Rubus parvifolius and $R$. coreanus (Rosaceae)," Genetic Resources and Crop Evolution, vol. 57, no. 3, pp. 431-441, 2010. 
[13] C. E. Finn, C. Kempler, and P. P. Moore, "Raspberry cultivars: what's new? What's succeeding? Where are breeding programs headed?," Acta Horticulturae, no. 777, pp. 33-40, 2008.

[14] G. B. Seymour, L. Østergaard, N. H. Chapman, S. Knapp, and C. Martin, "Fruit development and ripening," Annual Review of Plant Biology, vol. 64, no. 1, pp. 219-241, 2013.

[15] J. F. Sánchez-Sevilla, J. G. Vallarino, S. Osorio et al., "Gene expression atlas of fruit ripening and transcriptome assembly from RNA-seq data in octoploid strawberry (Fragaria $\times$ ananassa)," Scientific Reports, vol. 7, no. 1, article 13737, 2017.

[16] S. Zenoni, A. Ferrarini, E. Giacomelli et al., "Characterization of transcriptional complexity during berry development in Vitis vinifera using RNA-seq," Plant Physiology, vol. 152, no. 4, pp. 1787-1795, 2010.

[17] V. Gupta, A. D. Estrada, I. Blakley et al., "RNA-Seq analysis and annotation of a draft blueberry genome assembly identifies candidate genes involved in fruit ripening, biosynthesis of bioactive compounds, and stage-specific alternative splicing," GigaScience, vol. 4, no. 1, pp. 1-22, 2015.

[18] N. Fernandez-Pozo, Y. Zheng, S. I. Snyder et al., "The tomato expression atlas," Bioinformatics, vol. 33, no. 15, pp. 23972398, 2017.

[19] B. Langmead and S. L. Salzberg, "Fast gapped-read alignment with Bowtie 2," Nature Methods, vol. 9, no. 4, pp. 357-359, 2012.

[20] C. F. Williams, "Influence of parentage in species hybridization of raspberries," Proceedings, American Society for Horticultural Science, vol. 56, pp. 149-156, 1950.

[21] F. A. Simão, R. M. Waterhouse, P. Ioannidis, E. V. Kriventseva, and E. M. Zdobnov, "BUSCO: assessing genome assembly and annotation completeness with single-copy orthologs," Bioinformatics, vol. 31, no. 19, pp. 3210-3212, 2015.

[22] F. Musacchia, S. Basu, G. Petrosino, M. Salvemini, and R. Sanges, "Annocript: a flexible pipeline for the annotation of transcriptomes able to identify putative long noncoding RNAs," Bioinformatics, vol. 31, no. 13, pp. 2199-2201, 2015.

[23] R. T. Arrial, R. C. Togawa, and M. d. M. Brigido, "Screening non-coding RNAs in transcriptomes from neglected species using PORTRAIT: case study of the pathogenic fungus Paracoccidioides brasiliensis," BMC Bioinformatics, vol. 10, no. 1, p. 239, 2009.

[24] B. Li and C. N. Dewey, "RSEM: accurate transcript quantification from RNA-Seq data with or without a reference genome," BMC Bioinformatics, vol. 12, no. 1, p. 323, 2011.

[25] M. I. Love, W. Huber, and S. Anders, "Moderated estimation of fold change and dispersion for RNA-seq data with DESeq2," Genome Biology, vol. 15, no. 12, p. 550, 2014.

[26] M. D. Robinson, D. J. McCarthy, and G. K. Smyth, "edgeR: a bioconductor package for differential expression analysis of digital gene expression data," Bioinformatics, vol. 26, no. 1, pp. 139-140, 2010.

[27] M. E. Ritchie, B. Phipson, D. Wu et al., "limma powers differential expression analyses for RNA-sequencing and microarray studies," Nucleic Acids Research, vol. 43, no. 7, article e47, 2015.

[28] Q. Yu, A. Plotto, E. A. Baldwin et al., "Proteomic and metabolomic analyses provide insight into production of volatile and non-volatile flavor components in mandarin hybrid fruit," BMC Plant Biology, vol. 15, no. 1, p. 76, 2015.

[29] K. Klesk and M. Qian, "Preliminary aroma comparison of Marion (Rubus spp. hyb) and Evergreen (R. laciniatus L.) blackberries by dynamic headspace/OSME technique," Journal of Food Science, vol. 68, no. 2, pp. 697-700, 2003.

[30] M. Saladie, A. J. Matas, T. Isaacson et al., "A reevaluation of the key factors that influence tomato fruit softening and integrity," Plant Physiology, vol. 144, no. 2, pp. 1012-1028, 2007.

[31] A. Payasi, N. N. Mishra, A. L. S. Chaves, and R. Singh, "Biochemistry of fruit softening: an overview," Physiology and Molecular Biology of Plants, vol. 15, no. 2, pp. 103-113, 2009.

[32] S. Posé, J. A. García-Gago, N. Santiago-Doménech, F. PliegoAlfaro, M. A. Quesada, and J. A. Mercado, "Strawberry fruit softening: role of cell wall disassembly and its manipulation in transgenic plants," Genes, Genomes and Genomics, vol. 5, no. 1, pp. 40-48, 2011.

[33] D. Stewart, P. P. M. Iannetta, and H. V. Davies, "Ripeningrelated changes in raspberry cell wall composition and structure," Phytochemistry, vol. 56, no. 5, pp. 423-428, 2001.

[34] A. R. Vicente, C. Ortugno, A. L. T. Powell, L. Carl Greve, and J. M. Labavitch, "Temporal sequence of cell wall disassembly events in developing fruits. 1. Analysis of raspberry (Rubus idaeus)," Journal of Agricultural and Food Chemistry, vol. 55, no. 10, pp. 4119-4124, 2007.

[35] S. Heo, D. Y. Lee, H. K. Choi et al., "Metabolite fingerprinting of bokbunja (Rubus coreanus Miquel) by UPLC-qTOF-MS," Food Science and Biotechnology, vol. 20, no. 2, pp. 567-570, 2011.

[36] D. Y. Lee, S. Heo, S. G. Kim et al., "Metabolomic characterization of the region- and maturity-specificity of Rubus coreanus Miquel (Bokbunja)," Food Research International, vol. 54, no. 1, pp. 508-515, 2013.

[37] C. Feng, M. Chen, C. J. Xu et al., "Transcriptomic analysis of Chinese bayberry (Myrica rubra) fruit development and ripening using RNA-Seq," BMC Genomics, vol. 13, no. 1, p. 19, 2012.

[38] S. Miosic, J. Thill, M. Milosevic et al., "Dihydroflavonol 4reductase genes encode enzymes with contrasting substrate specificity and show divergent gene expression profiles in Fragaria species," PloS One, vol. 9, no. 11, article e112707, 2014.

[39] H. A. Daubeny and H. S. Pepin, "Variations among red raspberry cultivars and selections in susceptibility to the fruit rot causal organisms Botrytis cinerea and Rhizopus spp," Canadian Journal of Plant Science, vol. 54, no. 3, pp. 511516, 1974.

[40] E. Keep, V. H. Knight, and J. H. Parker, "Rubus coreanus as donor of resistance to cane diseases and mildew in red raspberry breeding," Euphytica, vol. 26, no. 2, pp. 505-510, 1977.

[41] H. K. Hall, K. E. Hummer, A. R. Jamieson, S. N. Jennings, and C. A. Weber, "Raspberry breeding and genetics," in Plant breeding reviews, J. Janick, Ed., vol. 32, pp. 148-169, John Wiley \& Sons, Inc., Hoboken, NJ, USA, 2009.

[42] H. K. Hall and C. Kempler, "Raspberry breeding," Fruit, Vegetable and Cereal Science and Biotechnology, vol. 5, Supplement 1, pp. 44-62, 2011.

[43] K. E. Hammond-Kosack and J. D. G. Jones, "Plant disease resistance genes," Annual Review of Plant Physiology and Plant Molecular Biology, vol. 48, no. 1, pp. 575-607, 1997.

[44] Z.-Q. Shao, J.-Y. Xue, P. Wu et al., "Large-scale analyses of angiosperm nucleotide-binding site-leucine-rich repeat genes reveal three anciently diverged classes with distinct evolutionary patterns," Plant Physiology, vol. 170, no. 4, pp. 20952109, 2016. 
[45] J. S. Moreira, R. G. Almeida, L. S. Tavares et al., "Identification of botryticidal proteins with similarity to NBS-LRR proteins in rosemary pepper (Lippia sidoides Cham.) flowers," Protein Journal, vol. 30, no. 1, pp. 32-38, 2011.

[46] Y. Jia, Y. Yuan, Y. Zhang, S. Yang, and X. Zhang, "Extreme expansion of NBS-encoding genes in Rosaceae," BMC Genetics, vol. 16, no. 1, p. 48, 2015.

[47] S. K. Samuelian, A. M. Baldo, J. A. Pattison, and C. A. Weber, "Isolation and linkage mapping of NBS-LRR resistance gene analogs in red raspberry (Rubus idaeus L.) and classification among 270 Rosaceae NBS-LRR genes," Tree Genetics \& Genomes, vol. 4, no. 4, pp. 881-896, 2008.

[48] L. Afanador-Kafuri, J. F. Mejía, A. González, and E. Álvarez, "Identifying and analyzing the diversity of resistance gene analogs in Colombian Rubus genotypes," Plant Disease, vol. 99, no. 7, pp. 994-1001, 2015.

[49] Y. Zhang, R. Xia, H. Kuang, and B. C. Meyers, "The diversification of plant NBS-LRR defense genes directs the evolution of microRNAs that target them," Molecular Biology and Evolution, vol. 33, no. 10, pp. 2692-2705, 2016.

[50] B. C. Meyers, M. Morgante, and R. W. Michelmore, “TIR-X and TIR-NBS proteins: two new families related to disease resistance TIR-NBS-LRR proteins encoded in Arabidopsis and other plant genomes," The Plant Journal, vol. 32, no. 1, pp. 77-92, 2002.

[51] H. Takahashi, J. Miller, Y. Nozaki et al., "RCY1, an Arabidopsis thaliana RPP8/HRT family resistance gene, conferring resistance to cucumber mosaic virus requires salicylic acid, ethylene and a novel signal transduction mechanism," The Plant Journal, vol. 32, no. 5, pp. 655-667, 2002.

[52] Q. Chen, H. Yu, H. Tang, and X. Wang, "Identification and expression analysis of genes involved in anthocyanin and proanthocyanidin biosynthesis in the fruit of blackberry," Scientia Horticulturae, vol. 141, pp. 61-68, 2012.

[53] A. M. Bolger, M. Lohse, and B. Usadel, "Trimmomatic: a flexible trimmer for Illumina sequence data," Bioinformatics, vol. 30, no. 15, pp. 2114-2120, 2014.

[54] H. S. Le, M. H. Schulz, B. M. McCauley, V. F. Hinman, and Z. Bar-Joseph, "Probabilistic error correction for RNA sequencing," Nucleic Acids Research, vol. 41, no. 10, article e109, 2013.

[55] C. Trapnell, A. Roberts, L. Goff et al., "Differential gene and transcript expression analysis of RNA-seq experiments with TopHat and Cufflinks," Nature Protocols, vol. 7, no. 3, pp. 562-578, 2012.

[56] B. J. Haas, A. Papanicolaou, M. Yassour et al., "De novo transcript sequence reconstruction from RNA-seq using the Trinity platform for reference generation and analysis," Nature Protocols, vol. 8, no. 8, pp. 1494-1512, 2013.

[57] W. Li and A. Godzik, "CD-HIT: a fast program for clustering and comparing large sets of protein or nucleotide sequences," Bioinformatics, vol. 22, no. 13, pp. 1658-1659, 2006.

[58] D. Gilbert, "Accurate \& complete gene construction with EvidentialGene," F1000Research, vol. 5, p. 1567, 2016.

[59] C. Xie, X. Mao, J. Huang et al., "KOBAS 2.0: a web server for annotation and identification of enriched pathways and diseases," Nucleic Acids Research, vol. 39, Supplement 2, pp. W316-W322, 2011.
[60] P. Arya, G. Kumar, V. Acharya, and A. K. Singh, "Genomewide identification and expression analysis of NBS-encoding genes in Malus $\times$ domestica and expansion of NBS genes family in Rosaceae," PLOS One, vol. 9, no. 9, article e107987, 2014.

[61] R. D. Finn, J. Clements, and S. R. Eddy, "HMMER web server: interactive sequence similarity searching," Nucleic Acids Research, vol. 39, Supplement_2, pp. W29-W37, 2011. 


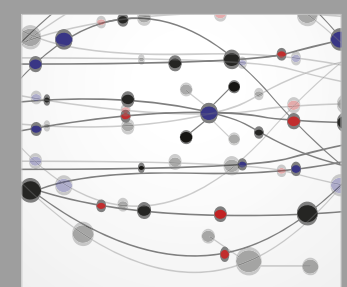

The Scientific World Journal
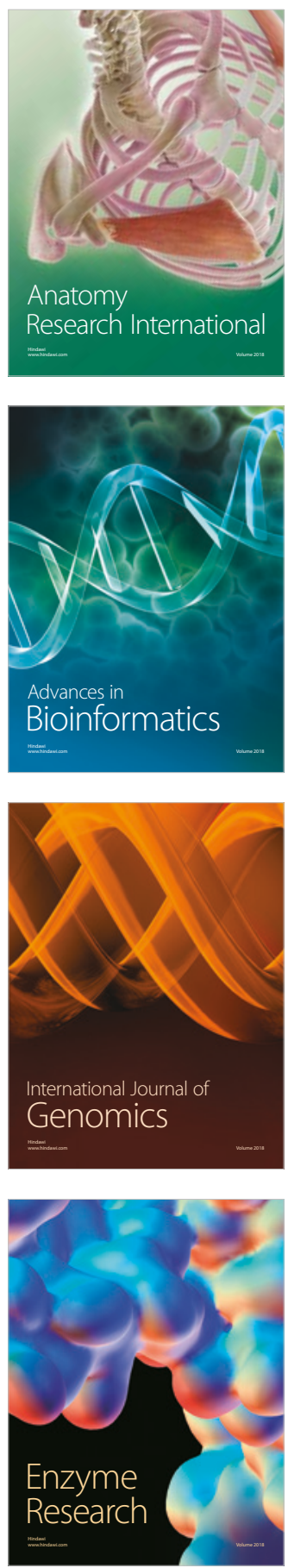
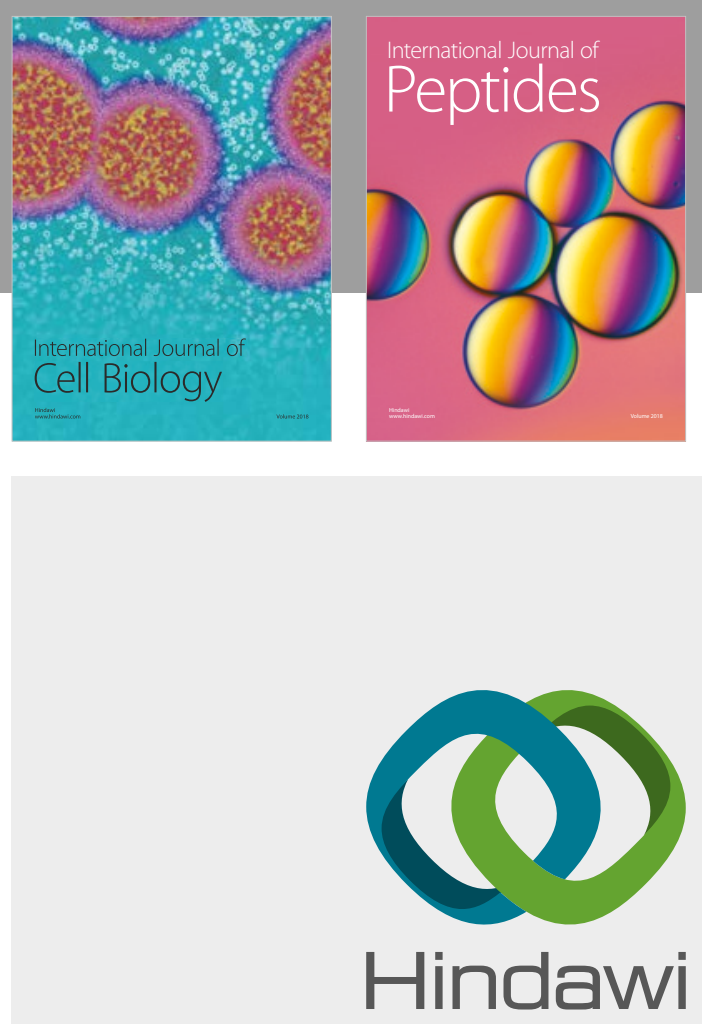

Submit your manuscripts at

www.hindawi.com
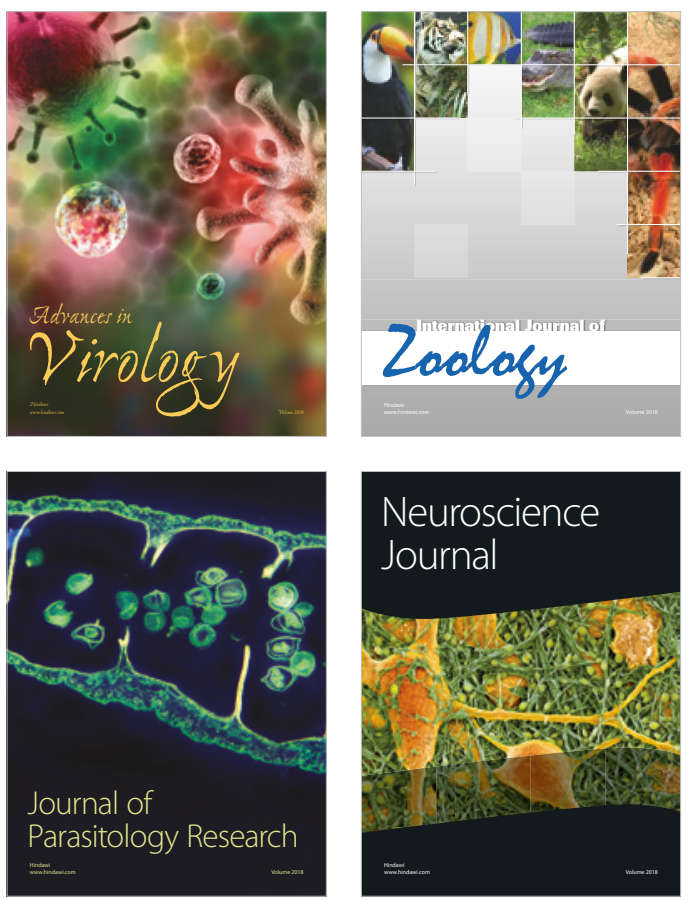
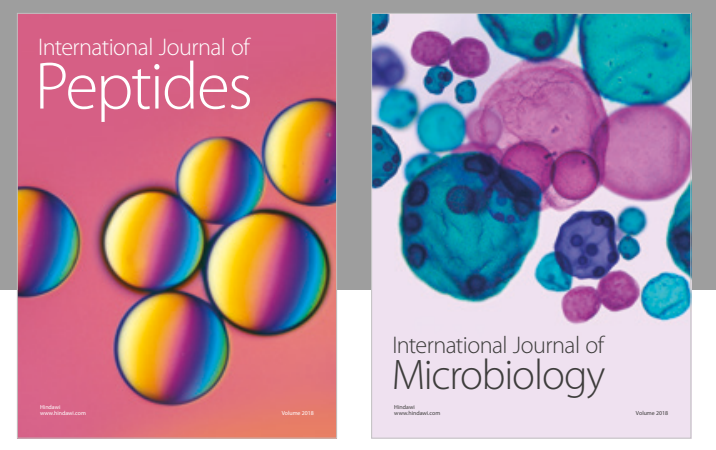

nternational Journal of Microbiology
Journal of
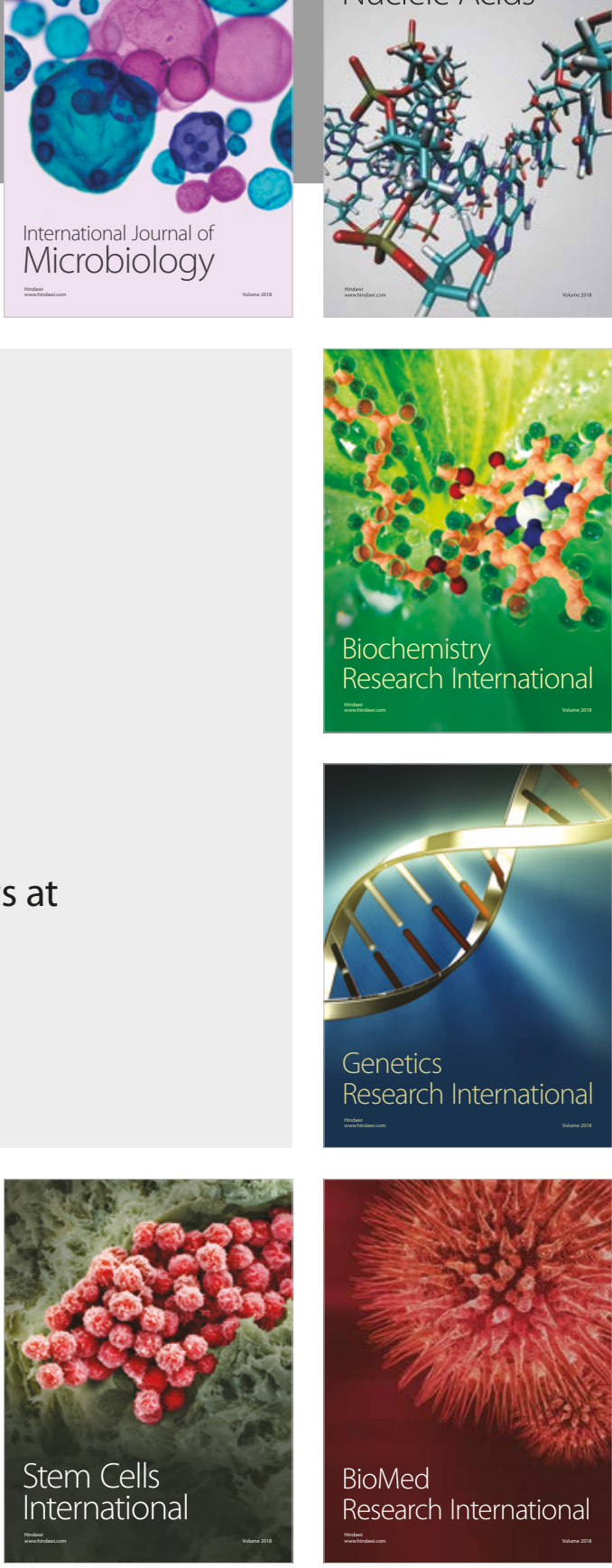
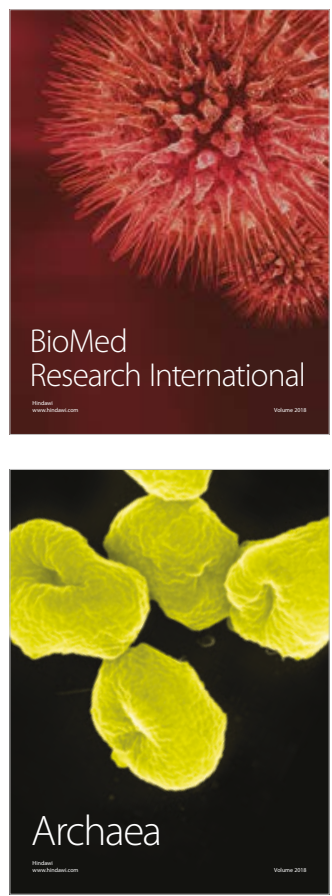